\title{
Des Français et de la langue française
}

\section{Yannick Lefranc}

\section{OpenEdition}

\section{Journals}

Édition électronique

URL : http://journals.openedition.org/esp/2765

DOI : 10.4000/esp.2765

ISSN : 2532-0319

\section{Éditeur}

Centre d'Information sur l'Éducation Bilingue et Plurilingue

\section{Édition imprimée}

Date de publication : 1 décembre 2013

Pagination : 63-68

ISSN : 1127-266X

\section{Référence électronique}

Yannick Lefranc, «Des Français et de la langue française », Éducation et sociétés plurilingues [En ligne], 35 | 2013, mis en ligne le 01 février 2020, consulté le 17 février 2021. URL : http:// journals.openedition.org/esp/2765; DOI : https://doi.org/10.4000/esp.2765 


\section{DES FRANÇAIS ET DE LA LANGUE FRANÇAISE (1)}

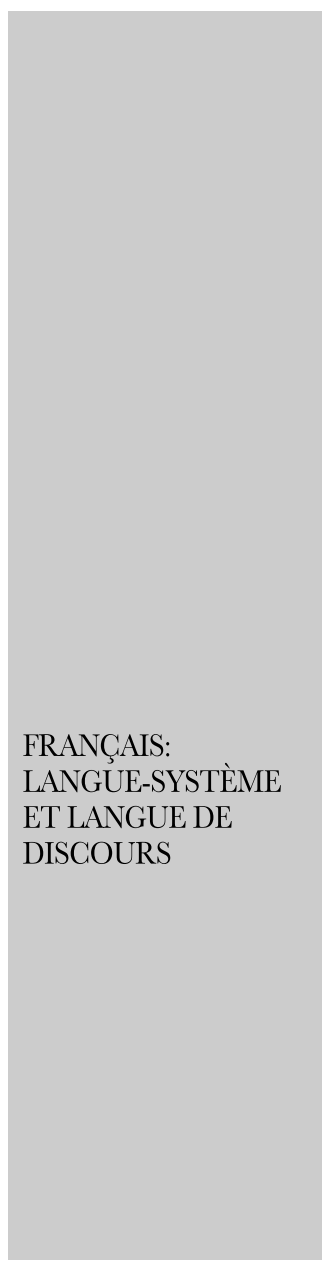

\section{Yannick LEFRANC}

$\mathrm{R}$ ythmés et modelés par la succession des lois sur la formation professionnelle et sur le séjour en France des migrants, les débats sur le droit à la langue soulèvent des questions de philosophie et de mesures politiques. D'un point de vue républicain, l'apprentissage et la maîtrise de la langue française, son appropriation, font partie des droits mais aussi des devoirs des habitants de France. Si l'on admet le principe «tous ceux qui vivent en France ont des droits et devoirs de citoyens». Ces derniers ont le devoir de se confronter aux énoncés et au système de la langue-culture en communiquant pour se mêler aux autres habitants - leurs semblables civiques - et ils ont le droit-pouvoir de se mêler en langue française des affaires de la cité.

Quelle importance et quelles caractéristiques donner aujourd'hui au «français langue nationale» (FLN), dans une Europe capitaliste où la mise en avant des diversités culturelles va de pair avec un renouvellement modernisé de la polarisation sociale? Les réflexions qui suivent avancent des définitions, des interrogations et des propositions qui cherchent à prendre en compte les nouvelles réalités françaises.

Comme les autres idiomes, le français est une langue-culture historique. C'est non seulement un «système», selon la conception réductrice de la grammaire scolaire et de la linguistique traditionnelle, mais c'est d'abord une langue trésor (2), composée de quantités de «discours en français» aux normes ou pas, légués par les siècles ou créés d'aujourd'hui, et que l'on réénonce et l'on incorpore plus ou moins partiellement et fidèlement.

Chaque citoyen parlant apprend et pratique le français au milieu d'un ensemble de formes verbales en circulation. Il en tire et mémorise une anthologie de discours et de formules (proverbes, expressions et phrases toutes faites). En même temps, il en extrait et associe des lexies (mots simples, expressions et autres formules), tandis qu'il en abstrait le systeme de distinctions et de combinaisons: il le construit et le met en schèmes.

Ge que j'appelle «le français» réfère à un ensemble hétérogène 
Des Français et de la langue française

Y. Lefranc

LE FRANÇAIS DE LA RÉPUBLIQUE, SES NORMES ET SES CONTRAINTES d'énoncés oraux et écrits circulants, des discours qui sont certes surveillés et contrôlés par les autorités et les agents des institutions chargés de les surnormaliser, mais qui sont aussi diversement régulés au fil des échanges formels ou informels entre les parleurs. Ainsi, la masse composite et changeante des paroles et des écrits en français déborde les régularités retravaillées de l'écrit standardisé: ce «français» que l'on apprend à oraliser par l'école et par les médias, avec ses énoncés modèles réglés, revus-relus, et corrigés.

À partir du français vivant instable et changeant, contre et au-dessus de son désordre plus ou moins organisé, les États de France ont institué et réinstitué une langue officielle, ou légitime: le français de la Nation, un idiome stabilisé et unifié par les conditionnements administratifs, juridiques, scolaires, littéraires, et médiatiques. Imposée à tous comme «le français», cette langue agit comme un modèle et fait pression sur les variations linguistiques orales et d'abord écrites. Le français surnormé exclut de son système et de son trésor bien des constructions et des mots propres aux pratiques langagières des classes populaires et moyennes inférieures des villes et des campagnes. Parmi les formes courantes du français ordinaire qui se disent et se reprennent, mais n'entrent pas dans le corpus restreint des écrits et des paroles du bon usage, académiquement et administrativement homologué, on remarquera beaucoup d'expressions et de constructions françaises créées accidentellement ou volontairement par des migrants, des ex-migrants, leurs enfants... et leurs petits-enfants.

Si c'est une langue internationale, et européenne, et s'il est proche de la langue des autres nations francophones, le français trésor-et-système qui s'apprend et se parle en France est avant tout la langue des habitants de cette République sociale et läque (suivant la constitution), la langue des « citoyens » qui y vivent et qui doivent pouvoir se l'approprier, quels que soient leur origine et leur milieu socioculturels, leur classe sociale ou leur communauté. C'est que les habitants ont intéret à l'apprendre, pour «se défendre dans la vie» individuellement et collectivement.

Certes, une telle vision correspond plus à un principe et à un idéal qu'à une réalité effective, mais c'est que la République sociale läque est un processus créatif, conflictuel, et à l'avenir incertain. De ce point de vue, la Révolution française, sociale et culturelle, ne serait pas terminée.

Langue de la communication publique, des rencontres et du mélange entre des habitants en accord ou en désaccord, le français doit l'emporter en France sur les autres langues du pays: les langues de la famille et les langues des médias, les langues régionales ou nationales. Pour autant, les citoyens doivent aussi pouvoir les apprendre, les pratiquer et 
Des Français et de la langue française

Y. Lefranc les diffuser.

C'est parier ici que la réalisation effective du droit à la langue nationale accroisse la capabilité des habitants jeunes ou vieux: i.e. qu'elle renforce leurs capacités affectivo-cognitives, conjuguées aux possibilités et aux libertés effectives d'exercer leurs capacités. Tout cela participe de ce processus de socialisation à la française, qui oblige les habitants à en accepter les normes et les principes pour exercer leurs pouvoirs politiques. Soulignons que, philosophiquement, les «valeurs républicaines» (les buts de vie déclarés bons pour tous) ne sont pas présentées comme surnaturelles mais sociohistoriques et politico-juridiques: elles dépendent d'une définition de l'intérêt général démocratiquement discutable.

Puisque les lois de la démocratie française l'emportent sur les lois des dieux et des déesses, sur celles des communautés (ou plutôt de leurs dirigeants), et sur celles des familles ou des ancêtres, la langue de la République - langue de Molière, Voltaire, Prévert, Césaire, et Cavanna - est aussi un espace linguistique où circulent des discours et des textes irrespectueux des croyances et des convictions des concitoyens (même républicaines), ce qui mécontentera ou choquera toujours quelqu'un.

Cependant, si la diversité conflictuelle des discours doit être reflétée par l'enseignement public et commun du français national, elle n'échappe pas à certaines prescriptions et proscriptions démocratiques. L'apprentissage du français comme langue des citoyens de France entre en contradiction avec des manières de faire, de parler et de penser ethnicistes, sexistes ou classistes présentes dans la société et incompatibles avec les principes, les idéaux et les pratiques universalistes de la République - une République dont les réalisations démocratiques sont précaires et insuffisantes.

Cette socialisation linguistique des habitants promeut également une culture de l'instruction et de l'esprit critique obligatoires qui revêt une dimension paradoxale de «contrainte émancipatrice». Des lois, des mesures et des appareils d'État imposent officiellement que, comme tout le monde, les non Français soient exposés aux discours de l'éducation et de la formation nationales. Que ces personnes, ou ceux qui parlent en leur nom (chefs de famille, patrons, directeurs de conscience, vedettes des médias, experts) le veuillent ou non. De Condorcet à aujourd'hui, la citoyenneté active dépend toujours de la diffusion d'une culture générale pour tous.

Dans ce cadre philosophique et politique que penser de l'institution d'un français langue de l'intégration (FLI) (3)? Le FLI aura sans doute des effets dissuasifs, et plusieurs bénéficiaires n'auront pas recours à ce «droit à la formation linguistique» s'il se traduit par des contraintes et des corvées administratives pour les migrants, avec des menaces et des 
Des Français et de la langue française

Y. Lefranc
DROIT À LA LANGUE FRANÇAISE ET DROIT AUX AUTRES LANGUES DE FRANCE sanctions pour les récalcitrants ou les méfiants (4). En tout cas, si l'on admet qu'une certaine connaissance et compétence de même qu'une pratique régulière du français sont indispensables pour obtenir la nationalité française, mais aussi pour être capable d'exercer des droits de citoyen avec ou sans la nationalité, une maitrise insuffisante du français standardisé, ou même le refus de l'apprendre ne doivent entraîner ni expulsion du territoire, ni exclusion, ni suppression de droits et de libertés.

Si elles sont démocratiquement élaborées et débattues, les formations et les certifications linguistiques devraient servir les intérêts de ces nouveaux citoyens, s'ils acquièrent la culture générale, civique et professionnelle nécessaire, et des pouvoirs politiques.

Cette acculturation en français impose également des obligations non négociables. Les citoyens doivent suivre les lois et certaines règles de vie créées au cours de luttes historiques, un ensemble de normes peu à peu construit contre des normes étatiques, religieuses, familiales et même entrepreneuriales. Aujourd'hui encore, les habitants doivent se battre pour défendre, consolider et enrichir les conquêtes sociales du passé français: droits à la santé, droits des salariés, droits des femmes, droits à l'instruction publique et à la culture générale, droits à la liberté de conscience et à l'expression publique de ses opinions - y compris critiques et satiriques.

Cette problématisation politique de la communication langagière demande d'admettre que toute vie en société comporte une dimension conflictuelle, et qu'il s'agit de la démocratiser. Une telle conception s'oppose à l'idéologie multiculturaliste, moraliste et crypto-religieuse véhiculée par tant de travaux éducatifs et linguistiques du Conseil de l'Europe, qui vantent la cohésion sociale en minorant ou en escamotant les rapports de forces, de domination et d'exploitation, de soumission et de lutte.

L'apprentissage du français, dit-on, doit tenir compte des langues premières des parleurs et les intégrer dans les cours de $\mathrm{FL}$ «M» et de FLS. Comment y parvenir si l'on en examine froidement les conditions de possibilité et de contrainte d'une France qui n'émerge pas en bon état de la crise mondiale? Comment financer ces formations, les organiser et les concrétiser?

En nous limitant aux principes, on conviendra cependant que le français, ses apprentissages, ses usages et son système-trésor doivent se renouveler pour s'adapter aux changements historiques d'une nation socio-économiquement et culturellement mondialisée. Mieux encore, s'il se veut républicain et social, l'enseignement actualisé du français associera les langues des migrants au FL «M», au FLS et au FLI. Conjuguant ses capacités-pouvoirs de communication et d'expression à 
Des Français et de la langue française

Y. LeFRANC

DU DROIT À LA PAROLE AU DROIT SUR LA LANGUE son «pouvoir de traduction» (Balibar, 1985), l'étudiant-stagiaire apprendra et pratiquera le français en s'accoutumant à faire des allers et retours entre sa/ses langue/s première/s et la langue légitime - articulée au français familier et populaire. En France, la langue nationale servirait à la traduction et à la reformulation des discours de ses citoyens. Le français légitime se montrerait enfin ouvert aux autres idiomes du pays. À condition que la République crée les emplois de formateurs-interprètes de français dont la population, la nation, a besoin.

Les institutions d'instruction et de culture ont à faire du français national une langue véritablement commune, rendue disponible et intelligible pour tous: une langue communale - comme on parle de terrains communaux. Il s'agit donc à la fois de faire advenir et d'accroitre les pouvoirs d'expression, de communication et de traduction en français de tous, mais également de créer des règles et des institutions démocratiques pour que chacun ait réellement la possibilité de se mêler aux autres habitants en langue française. Y compris en employant des formulations et des tournures influencées par d'autres langues et par d'autres variétés de français. Elles renouvelleront et elles enrichiront la langue de tous.

Pour devenir réellement démocratique, le français normalisé sera remis sur le métier, et l'on s'inspirera de la souplesse et de la créativité du français populaire, comme de celles de l'anglo-américain, officiel ou courant. En comparaison avec l'anglais national-international, la langue française «correcte» apparait actuellement comme une langue tout à la fois appauvrie et corsetée. Un idiome dont les nouveautés verbales sont des calques de l'anglais médiatique et managérial de la mondialisation. Et si l'on remettait le bonnet rouge au dictionnaire (Victor Hugo), en enrichissant le français des mots et des expressions des langues régionales, et des créations des_parleurs des classes populaires et moyennes de toutes origines?

La lutte sociale et politique qui promeut le droit à la langue pour tous, pour tous les habitants (Viviane Forester), pour tous les icitiens (Jamel Debbouze), qu'ils soient migrants, ex-migrants ou non migrants, pourrait jouer un rôle de révélateur, et lever du même coup le voile sur la dépossession de la langue nationale que vivent la majorité des citoyens de France - pourtant scolarisés et médiatisés. Le droit et le devoir de s'approprier le français standardisé écrit-oralisé va de pair avec un droit sur la langue qui doit échapper au monopole des dirigeants et des experts économiques, étatiques, et académiques.

Si le droit à et sur la langue française et si le devoir de connaitre et de 
Des Français et de la langue française

Y. LeFrANC

\section{CAPABILITÉ ET CONDITIONS DE POSSIBILITÉ}

\section{RÉFÉRENCES}

\section{NOTES}

(1) Une première version de cet article est parue sous le titre «De la langue française et de ses habitants associés. Remarques et propositions sur les droits, devoirs et pouvoirs linguistiques des citoyens parlants», Savoirs et Formation, Revue de l'AEFTI n 82, décembre 2011.

(2) J'interprète ici librement le terme de Saussure (Godel, 1957) que je redéfinis comme une configuration langagière où les formes verbales mémorisées par les sujets (leurs «trésors» au sens de Saussure) interagissent avec les discours circulants: avec le «trésor» des formes discursives produites par les locuteurs en communication sociale. Cette vision de la langue comme langue-de-discours ou discours-de-la-langue s'inspire de Meschonnic (1997).

(3) Depuis 2011, les étrangers non européens candidats à la nationalité française doivent prouver qu'ils ont un niveau de compétence orale du français qui équivaut au niveau B1 du CECR européen. Le FLI se distingue cependant du FLE par son contenu et ses finalités civiques et politiques.

Sur le phénomène du non-recours aux droits et aux services sociaux, voir le site: http://www.inegalites.fr/spip.php?article1495 